\title{
Performance Analysis of Different Indoor Ultra Wide Band Channel Indicators
}

\author{
Antonio Sorrentino ${ }^{1}$, Lorenzo Mucchi ${ }^{2}$, Sergio Cappa ${ }^{1}$, Maurizio Migliaccio ${ }^{1}$ \\ ${ }^{1}$ Università degli Studi di Napoli Parthenope, Dipartimento di Ingegneria, \\ Centro Direzionale di Napoli, Isola C4, 80143 Napoli, Italy. \\ ${ }^{2}$ Dept. of Information Engineering, University of Florence, Italy \\ *corresponding author, E-mail: asorrentino@uniparthenope.it
}

\begin{abstract}
In this paper, performance of different indicators for the profiling of Ultra Wide Band (UWB) wireless propagation channel are analyzed. In particular, the $\gamma$-indicator and the kurtosis index $k$ are compared in terms of the standard error. In order to improve the accuracy in the kurtosis case, results of the bootstrap error procedure are also accomplished. Further, an evaluation on the computational time needed for error estimation, is also provided. The comparison is made according to a real set of data derived from UWB measurement campaign accomplished within a modern laboratory/office building in which the two above mentioned indicators have been evaluated.
\end{abstract}

\section{Introduction}

With the increasing of wireless communication systems several studies on the modeling of the propagation channels have been accomplished. Particular attention has been given to the Ultra Wide Band (UWB) systems for the larger bandwidth compared to the current wireless systems (such as Bluetooth, WiFi, GSM/UMTS), and, above all, for the low-power/high data rate technology. UWB is becoming widespread in wireless communications area and the research on UWB applications is getting more attention in the industry. The great advantages of UWB signals are given by their wall and other object penetration, allowing the UWB system to operate also in severe NLOS scenarios. These potentials, together with low cost trans-receiver, low transit power and low interference characteristics, make the UWB technology an excellent candidate for a wide range of measurement applications, such as wireless propagation modeling [1-3], localization for indoor and outdoor positioning [4-6] and short range applications [7-8]. In all the above-mentioned applications, it is important to know the propagation channel to maximize the system performance.

The system performance maximization starts from the development of electromagnetic models in which the quality of wireless communications services could be identified by means of simple parameters. By searching these model parameters to match different channel characteristics, the channel model can be established. Many efforts and resources have been devoted in the last few years to characterize and model the UWB propagation channels, and several parameters have been proposed [1, 3]. In [3], based on the physical K-Generalized model, the $\gamma$ parameter developed for observation of synthetic aperture radar (SAR) data and for studying the reverberating chamber propagation channel profiles [9-10], has been proposed to physically discriminate Line of Sight (LOS) and non-LOS (NLOS) UWB propagation conditions. In [1, 11], the kurtosis parameter $k$ has been proposed to identify the typology of the link between the transmitting and the receiving antennas. Both of the two above mentioned parameters, i.e., the kurtosis $k$ and the gamma-indicator $\gamma$, have been used in the past to discriminate channel profiles, but no comparison has been accomplished to establish which indicator has the best performance. A missing of real-time information on the channel condition (LOS/NLOS) can provide a severe decrease of the performance of an UWB system, especially in some applications such as localization for indoor positioning, where the real-time knowledge of the propagation channel profile is a very challenging task for the correct system characterization.

In this paper, the two indicators, $k$ and $\gamma$, are compared to establish which one has the best performance in discriminating indoor LOS/NLOS propagation conditions. The metric for comparing the performance is the standard error, hereinafter-called $S E$ [12]. Since the traditional standard errors tend to under or overestimate true errors, the bootstrap method for standard error estimation, is also implemented [13]. Finally, the computational time for the above-mentioned indicators is also evaluated. It is important to note that, since this analysis is focused on improving the accuracy of the channel estimation, the Rice factor $R$ is not considered here as a direct candidate parameter to identify LOS/NLOS condition. Although $R$ is one of the most reliable index when a strong direct link component is present, it cannot discriminate small but consistent direct link component according to the minimum requirements for specifically UWB propagation channels $[1,3]$.

The comparison is made according to a real set of data derived from UWB measurement campaign accomplished within a modern laboratory/office building in which the two above mentioned indicators have been evaluated in LOS/NLOS propagation conditions.

The paper is structured as follows: in the section 2 an electromagnetic analysis of the employed UWB indicators is accomplished; in section 3 a meaningful set of 
experimental results is shown and discussed. In sections 4 and 5, the errors of the two indicators used for UWB channels discriminations are described and compared. Finally, in section 6, conclusions are drawn.

\section{Electromagnetic Theory of UWB Indicators}

In this section, a brief but exhaustive analysis of the employed UWB indicators is accomplished. Starting from $n$ time series of data, i.e., of the electromagnetic field at the receiving antenna, the physical rationale of the two indicators is summarized.

\section{1. $\gamma$ - indicator}

The presence of the direct link within a generic UWB propagation channel can be characterized by analyzing the amplitude/power of the received electromagnetic field. In particular, the electromagnetic field within an UWB wireless propagation channel can be described by the Generalized K (GK) model [3] that is given by

$$
\begin{aligned}
p\left(E^{2}\right) & =\frac{2 \alpha}{\Gamma(\alpha) \eta^{\alpha+1}}\left(\frac{\alpha}{1+\frac{v^{2}}{4 \alpha}}\right)^{\frac{\alpha-1}{2}} . \\
& \cdot E^{2 \frac{\alpha-1}{2}} I_{0}\left(\frac{v}{\eta} \sqrt{E^{2}}\right) K_{\alpha-1}\left\{\frac{2}{\eta}\left[\left(1+\frac{v^{2}}{4 \alpha}\right) \alpha E^{2}\right]\right\}
\end{aligned}
$$

where $E^{2}$ is the power of the electromagnetic field, $\alpha, \eta$ and $v$ are the distribution parameters, $\Gamma(\cdot)$ is the Eulerian Gamma function, $K_{\alpha-1}(\cdot)$ is the modified Bessel function of the second kind of order $\alpha-1$ and $I_{0}(\cdot)$ is the first kind zeroorder modified Bessel function. The GK is a three-parameter distribution that embodies in a unitary formulation the statistic models used to characterize different propagation channel $[3,9]$. The three real and non-negative parameters, $\alpha, \eta$ and $v$, dictate the GK distribution behaviour [3].

For the purpose of this study only $\eta$ and $v$ (hereinafter termed as GK parameters) are accounted for, since they have been found to exhibit a pronounced sensitivity to the presence of a coherent component in the received field [3]. If a coherent component in the received field is contemplated, (1) degenerates in a Rice distribution, i.e. [3]

$$
p\left(E^{2}\right)=\frac{1}{\eta^{2}} \exp \left(-\frac{v^{2}}{4}\right) \exp \left(-\frac{E^{2}}{\eta^{2}}\right) I_{0}\left(\frac{v \sqrt{E^{2}}}{\eta}\right)
$$

where only $\eta$ and $v$ are present [3]. If one consider the $R$ factor, i.e. the coherent to incoherent power ratio, (2) can be rewritten as follows

$p\left(E^{2}\right)=\frac{1+R}{\overline{E^{2}}} \exp \left[-\left(R+\frac{E^{2}}{\overline{E^{2}}}(1+R)\right)\right] I_{0}\left(2 \sqrt{\frac{E^{2}}{\overline{E^{2}}} R(R+1)}\right)$ (3)

According to what above mentioned and comparing (2) to (3), the $\gamma$-indicator is obtained as a proper combination of the GK parameters given in terms of $R$ [3]

$$
\gamma=\left(\frac{v}{\eta}\right)^{2}=\left(\frac{2 \sqrt{R}}{\sqrt{\frac{\bar{E}^{2}}{R+1}}}\right)^{2}
$$

where $\bar{E}^{2}$ is the mean power of the received field and $R$ is the Rice factor. It is important to note that the $\gamma$-indicator given by (4), depends on both $R$ and the mean power of the received field [9]. To be independent by the channel parameter, i.e., the mean power of the received field, a normalization can be accomplished by dividing $\bar{E}^{2}$ for the maximum received power [9]

$$
\beta=\frac{\bar{E}^{2}}{E_{\max }^{2}}
$$

The $\beta$ parameter is the inverse of the peak-to-average power-ratio (PAPR) and, in the real-life cases, it is always smaller than one. The PAPR parameter has been largely discussed in literature [14]. As matter of fact, with the PAPR reduction techniques recently developed, PAPR values typically range from 3 to $7 \mathrm{~dB}$ for UWB applications that correspond to values of $\beta$ that range from about 0.5 to 0.2 . By using (5) in (4), a modified expression of the $\gamma$ indicator can be obtained

$$
\gamma_{\bmod }=\left(\frac{2 \sqrt{R}}{\sqrt{\frac{\bar{E}^{2}}{E_{\max }^{2}} \frac{1}{R+1}}}\right)^{2}=\left(\frac{2 \sqrt{R}}{\sqrt{\frac{\beta}{R+1}}}\right)^{2}
$$

It is now important to read the $\gamma_{\text {mod }}$ behaviour given by (6) in terms of $R$ parameter. When $R \cong 0$ (generally $R<0.1$ ) the $\gamma_{\text {mod }}$ given by (6) is always smaller than one. Otherwise, (generally when $R \geq 0.1$ ) $\gamma_{\text {mod }}$ values are greater than one. Accordingly, this calls for two non-overlapped regions, i.e., [9]

$$
\gamma_{\text {mod }} \geq 1
$$

when a small but consistent line of sight component, i.e., when a LOS condition is in place, and

$$
\gamma_{\text {mod }}<1
$$

when the NLOS conditions applies. It is important to note that the $\gamma_{\text {mod }}$ index exhibits a much greater sensitivity with respect to the $R$ factor since it is able to discriminate the presence of small but consistent LOS components, that are considered negligible with the Rice factor analysis. Thus, $\gamma_{\text {mod }}$ is useful to obtain a reliable and high throughput link in the employed propagation channels.

\subsection{B. Kurtosis}

The kurtosis, $k$, is a low-complexity statistical parameter that indicates the fourth order moment of the received signal amplitude. The kurtosis has been already used for identifying the UWB channel profile [1] and for the reverberating chamber propagation channel profiles [11]. By 
considering eq. (3), kurtosis is mathematically defined as follows $[15,16]$

$$
k=\frac{1}{\sigma^{4}} \frac{\sum_{i}\left(E_{i}-\bar{E}\right)^{4}}{n}
$$

where $\sigma$ and $\bar{E}$ are the standard deviation, the mean and the $i$ th received field $E_{i}$, respectively, and $n$ is the dimension of the acquired time series. The kurtosis index $k$ is supposed to be high in case of LOS conditions while it has low values under NLOS conditions. The parameter $k$ does not need any application of estimation algorithms on the received field because it is calculated directly by using the received field samples. This makes $k$ really simple to manage and the identification process easy and quick. A limitation on the use of this indicators is given by the not exactly definition of a threshold for the different profile (LOS/NLOS). In other words, the $k$ analysis does not provide analytically a physical threshold for distinguishing the different typologies of channel profile in non-overlapped classes; only basing on the knowledge of the environment typology, $k$ can determine, in a quick way, if the LOS or NLOS channel profile is in place.

\section{Experimental Results}

In this section some meaningful results are shown and discussed. The experimental data are obtained from indoor UWB experiments (see Fig. 1). In [1], experimental results were carried out in a modern indoor University laboratory; the considered scenario is shown in Fig. 1 (taken from [1] with permission of the authors). The data is acquired with a sampling rate equal to $20.48 \mathrm{GHz}$, i.e., a sampling time of 48.83 ps. Impulse response measurements are gathered over a 7 × 7 square $15 \mathrm{~cm}$ spaced measurement grid, covering about a $90 \mathrm{~cm}$ x $90 \mathrm{~cm}$ surface (Fig. 1). Two UWB channel profiles have been accomplished: the line-of-sight (LOS) and the non-LOS (NLOS) channels. The $k$ and $\gamma$ values are evaluated and the results are reported in Table I for the LOS and NLOS cases, respectively. The LOS cases correspond to UWB propagation environments Room F1 and F2 in Fig. 1 (called LOS1 and LOS2 in Table I, respectively), in which the transmitting and the receiving antennas are placed in the same room with distances $9.5 \mathrm{~m}$ and $5.5 \mathrm{~m}$, respectively. The NLOS cases are relative to different rooms in which the transmitting and the receiving antennas are placed. In these conditions, at least one wall that totally obstructs the first Fresnel zone is present between the antennas, i.e., NLOS channels are accomplished. In particular, the distance between the transmitting and the receiving antennas is equal to $10 \mathrm{~m}$ and $17 \mathrm{~m}$, that corresponds to Room $\mathrm{H}$ and $\mathrm{B}$ in Fig. 1 , (NLOS1 and NLOS2 in Table I, respectively). The $k$ and $\gamma$ values are in agreement with the theory reported in section II and they are listed in Table I. In particular, in the LOS cases, the $k$ values are higher than the corresponding ones for the NLOS cases, while the obtained values are greater than the physical threshold for the $\gamma$ parameter (see Table I). It is important to note that although several studies have been accomplished with the $k$ index, no physical thresholds for discriminating the LOS and NLOS channel profiles is analytically provided. Hence, it is very challenging to discriminate the correct channel profile if there is no a priori knowledge of the environment. By a quantitative analysis the comparison of the two indicators used for distinguishing LOS and NLOS scenarios shows that $\gamma$ exhibits a greater granularity with respect to $k$ in discriminating LOS conditions. Concerning the computational time-consuming, $k$ is faster than $\gamma$. As matter of fact, by comparing the two algorithm with a $3.8 \mathrm{GHz}$ quad-core $7^{\text {th }}$ generation intel core i7 processor with $16 \mathrm{~GB} 1333 \mathrm{MHz}$ memory, the estimation time of $k$ parameter is equal to $1.2 \mathrm{~s}$, smaller than $3.0 \mathrm{~s}$ of the corresponding $\gamma$ parameter. Although the difference between the two parameters in terms of computational time in evaluating the LOS/NLOS condition can be considered negligible (smaller than $2 \mathrm{~s}$ ), it can be important in applications as localization and tracking. In fact, indoor accurate positioning requires to know if the distance estimation is made under LOS or NLOS since the corresponding bias is significantly different. If changes from LOS to NLOS conditions are not quickly detected the error can affect the estimated distance and decrease the accuracy of the indoor positioning.

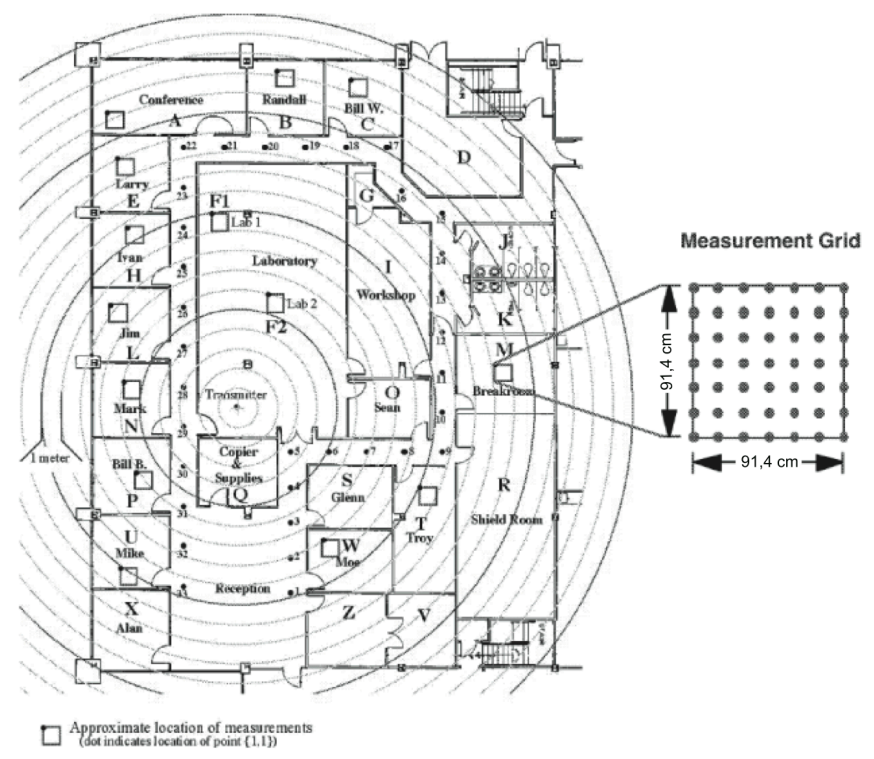

Figure 1: Scheck of the office/building used for UWB measurements, taken from [1] with permission of the authors

All those localization-based applications, which require accurate indoor positioning, could suffer of a 2 sec larger latency. In summary, from the experiments it follows that

- LOS and NLOS channel profiles are not overlapped both in the $\gamma$ than in the $k$ cases. A physical threshold for discriminating the two channel profiles is defined in the $\gamma$ - case but no threshold in the $k$ case is given. 
- From a computational time point of view performance of $k$ are better than the corresponding one of the $\gamma$-case. This is especially worth in those all localization-based applications.

Table 1: $v, \eta, \beta, \gamma$ and $k$ values for LOS/NLOS scenarios.

\begin{tabular}{cccccc}
\hline Channel & $v$ & $\eta$ & $\beta$ & $\gamma$ & $k$ \\
Profile & & & & & \\
\hline LOS1 & 9.78 & 0.03 & 0.198 & 84962.18 & 19.60 \\
LOS2 & 7.72 & 0.04 & 0.201 & 28167.80 & 39.93 \\
NLOS1 & 0.08 & 0.24 & 0.193 & 0.27 & 6.95 \\
NLOS2 & 0.06 & 0.28 & 0.191 & 0.12 & 4.85 \\
\hline
\end{tabular}

\section{Standard Error}

The focus of this section is to evaluate the standard error occurred when $\gamma$ or $k$ parameters are used to identify the channel LOS/NLOS conditions. The Standard Error, $S E$, is a measure of the accuracy with which the measured parameter represents the true value and is expressed as a number [12]. The $S E$ is defined as the standard deviation of the statistic parameter under evaluation, weighted for the sample size $n$. If one calls $S E_{k}$ the standard error in the case of $k$ parameter, it is given by [12]

$$
S E_{k}=2 \sqrt{\frac{6 n(n-2)(n-3)}{(n+1)^{2}(n+5)(n+3)}}
$$

where $n$ is the sample size. For $n$ large (usually greater than $40), S E_{k}$ is equal to

$$
S E_{k} \cong \frac{5}{\sqrt{n}}
$$

For the $\gamma$-index, it can be noted that the standard error of $\gamma$, $S E_{\gamma}$ is related to the $S E$ of the $\eta$ and $v$ parameters. By (6), it follows that [3]

$$
\begin{gathered}
\eta=\sqrt{\frac{\beta}{R+1}} \\
v=2 \sqrt{R}
\end{gathered}
$$

Hence, the $S E$ is proportional and inversely proportional to the square root of $R$ for $v$ and $\eta$ parameters, respectively [3]. $R$ is given by the power ratio of the direct link between the transmitting and the receiving antenna to the replicas of the transmitted signal, i.e.

$$
R=\frac{\bar{E}^{2}}{2 \sigma_{E}^{2}}
$$

where $\bar{E}^{2}$ and $\sigma_{E}^{2}$ are the power of the mean and the variance of the received field, respectively. According to the standard errors of the mean and the variance, $S E_{\bar{E}}$ and $S E_{\sigma_{E}^{2}}$ respectively, one obtains [12]

$$
S E_{\bar{E}}=\sigma_{E} / \sqrt{n}
$$

and

$$
S E_{\sigma_{E}^{2}}=\sqrt{\frac{2}{n-1}} \sigma_{E}^{2}
$$

Hence, the $S E$ of the $R$ factor is evaluated as [12]

$$
S E_{R}=\sqrt{\frac{n-1}{8 n^{2}}} \cong \frac{1}{2 \sqrt{2 n}}
$$

where $\cong$ means for $n$ large (usually $n>40$ ). According to (12)-(16) the $S E_{\gamma}$ for $n$ large is given by

$$
S E_{\gamma} \cong \frac{\sqrt{2 n}}{\beta n} \cong \frac{1}{\beta} \sqrt{\frac{2}{n}}
$$

A comparison between the standard error of both $\gamma$ and $k$ parameters is shown in Fig.2. It is important to note that, according to the literature and by considering the UWB indoor data of section $3, \beta$ values equal about to 0.2 are in place. The standard errors are reported in function of the number of samples used for the simulation. It can be noted that the two errors have the same trend although the $S E_{k}$ (dotted blue line) is always smaller than the corresponding one of the $\gamma$-case (continuous red line). As matter of fact, for small sample sizes $(n \leq 40)$ the gap between the two errors is 0.5 that decreases up to 0.1 for $n>40$, see Fig. 2 .

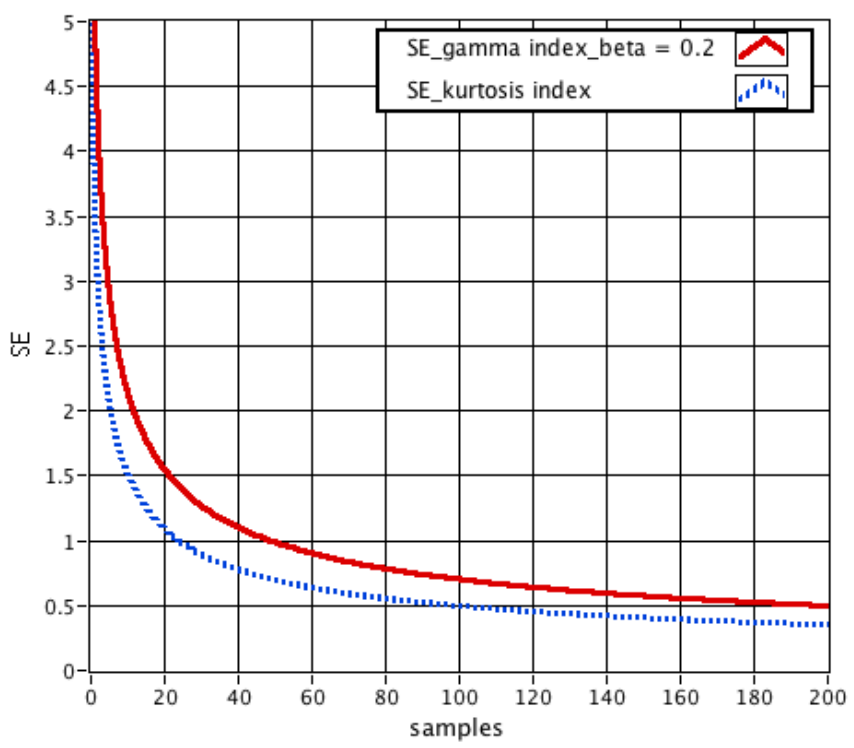

Figure 2: Comparison between $S E_{k}$ and $S E_{\gamma}$ evaluated for different value of $\beta$ parameter. 


\section{Bootstrap Error}

The standard error given by (11) in the kurtosis case, is function only of the number of sample $n$ and it does not depend on the distribution shape. In order to improve the statistical accuracy of the estimated parameters, such as the kurtosis, a method that takes into consideration also the distribution is needed. Bootstrap is one of the nonparametric techniques for statistical inference that are called resampling method [13]. The bootstrap method is based on the random resampling of the original data [13]. In other words, the bootstrap method involves repeatedly drawing random sample from the original data with replacement. For instance, if the original data set is $e=\left(e_{1}, e_{2}, \ldots, e_{7}\right)$ with $n=$ 7 and by taking $m$ samples of the same size $n$ as the original samples with replacement from the original sample, a new vector $e^{*}$ is obtained. This new vector of $m$-size is the first bootstrap sample. This process of the random resampling with replacement must be made $B$ times where typical values for $B$ range from 50 to 200 for standard error estimation [13]. In Fig. 3 a sketch of bootstrap error schematization is shown.

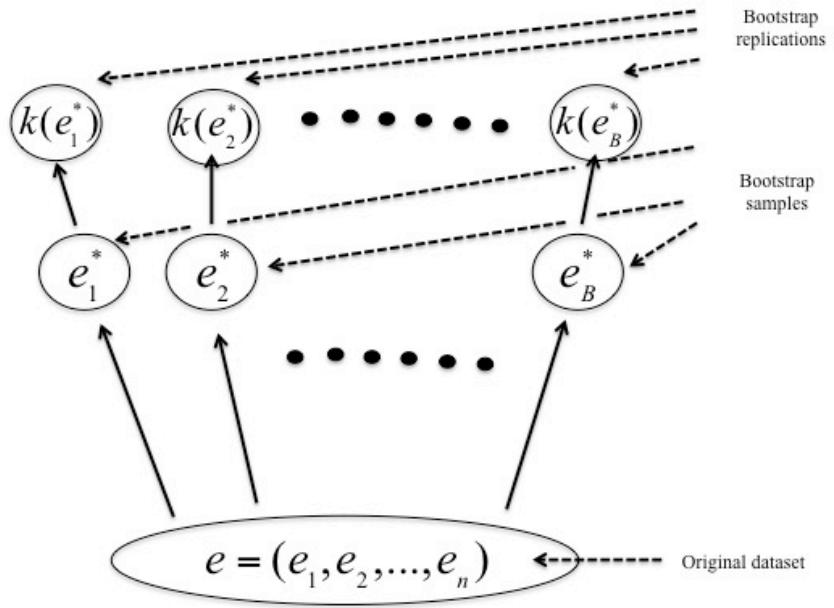

Figure 3: Bootstrap error method schematization

After the resampling with replacement to each bootstrap samples is in place, bootstrap replications $k\left(e_{B}\right)$ are obtained by calculating the value of statistics $k$ evaluated for $e^{*}$. Let us call $k\left(e_{B}^{*}\right)$ these bootstrap replications since the kurtosis, $k$, is the evaluated statistic. Hence, for each replications $k\left(e_{B}^{*}\right)$ the bootstrap standard error are estimated as follow [13]

$$
S E_{b o o t}(\cdot)=\left\{\frac{\sum_{b=1}^{B}\left[k\left(e_{b}^{*}\right)-\mu_{e_{b}^{*}}\right]^{2}}{B-1}\right\}^{1 / 2}
$$

where

$$
\mu_{e_{b}^{*}}(\cdot)=\frac{\sum_{b=1}^{B} k\left(e_{b}^{*}\right)}{B}
$$

is the mean of the bootstrap samples. In Fig. 4 a comparison among the bootstrap error, in the case of the kurtosis, and the standard error of $\gamma$ - and of $k$-indicator, is shown. The bootstrap error (black line) is higher than the corresponding standard error in the case of $\gamma$ - the and $k$ - indicator. It must be noted that $k$ evaluation involves the residual to the 4 th power and hence, it is influenced by extreme values that can distort the final results. The approach to use random bootstrap replication can avoid possible bias (due to the extreme distribution values) in the error analysis that can degree the accuracy of the method [13]. Finally, by comparing the two algorithm with a $3.8 \mathrm{GHz}$ quad-core $7^{\text {th }}$ generation intel core i7 processor with $16 \mathrm{~GB} 1333 \mathrm{MHz}$ memory, the bootstrap algorithm has about $2 \mathrm{~s}$ processing delay with respect to the $S E$ algorithm for the $\gamma$-case.

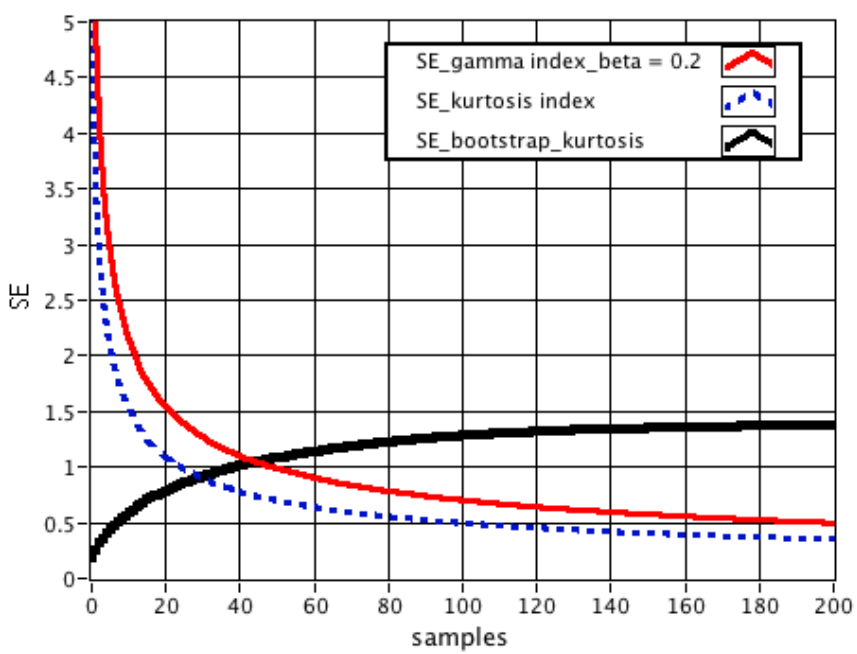

Figure 4: Comparison among $S E_{k}, S E_{\gamma}$ and the $S E$ evaluated by using the bootstrap method simulation.

In summary, by analyzing the obtained results, it follows that:

- the kurtosis procedure is really fast since it does not require high complexity evaluation with respect to $\gamma$ procedure where an estimation process of $\beta$ and $R$ are needed before its application;

- If the $S E$ error is evaluated, both indicators show a standard error always small; in particular the $S E$ of $k$ is always smaller than the corresponding one in the $\gamma$ cases;

- to further improve the accuracy of $S E$, a bootstrap error technique must be employed. Although an improved accuracy is pointed out, the bootstrap error technique calls for a slower $k$ procedure with respect to $\gamma$ standard error. In conclusions, the evaluation of the error corresponding to $\gamma$ indicator when the number of samples is large (usually greater than 40) is faster and gives best performance in discriminating LOS/NLOS propagation channels with respect to the corresponding one obtained by bootstrap error procedure in the $k$ case. 


\section{Conclusions}

In this paper, a comparison between $\gamma$ and $k$ indicators for defining UWB channel profiles, has been accomplished in terms of $S E$. The comparison has been developed according to experimental data gathered within a modern laboratory/office building in which the channel profiles (LOS/NLOS) have been obtained. A qualitative and a quantitative analysis show that:

- $\quad \gamma$ and the $k$ parameters values are in agreement with the expectation. The two indicator correctly distinguish in two non-overlapped value regions the LOS/NLOS channel profiles;

- $\quad \gamma$ exhibits a physical threshold for distinguishing LOS/NLOS profiles that is not present in the $k$ case;

- although $k$ has a computational time smaller that the corresponding $\gamma$ case, a bootstrap procedure is needed in the kurtosis case [13] making $k$ implementation longer than the corresponding $\gamma$. In other words, the computational time of the $S E_{\gamma}$ are smaller with respect to the corresponding bootstrap error technique applied in the $k$ case;

- Finally, the $\gamma$ error is smaller with respect to the bootstrap error applied in the $k$ case when the number of samples are large (usually greater than 40).

\section{References}

[1] L. Mucchi, P. Marcocci, "A New Parameter for UWB Indoor Channel Profile Identification", IEEE Trans. Wireless Communication, vol.8, no.4, pp.1597-1602, 2009.

[2] T. Santos, F. Tufvesson, A. F. Molish, "Modeling the ultra-wideband outdoor channel: model specification and validation", IEEE Trans. Wirel. Commun., vol.9, no.6, pp. 1987-1997, 2010.

[3] L. Mucchi, A. Sorrentino, A. Carpini, M. Migliaccio, G. Ferrara, "A Physically-Based Indicator for Identifying UWB Indoor Channel Condition", IET Microwave, Antennas \& Propagation, 2014

[4] J. Xu, M. Ma, L. Law, "Performance of time-differenceof-arrival ultra wideband indoor localization", IET Science, Measurement \& Technology”, vol. 5, no. 2, pp. 46-53, 2011.

[5] C. Zhang, M. J. Kuhn, B. C. Merkl, A. E. Fathy, M. R. Mahfouz, "Real-time Noncoherent UWB positioning radar with millimiter rance accuracy: theoory and experiment", IEEE Transaction on Microwave Theory and Techniques, vol. 58, no. 1, pp. 9-20, 2010.
[6] G. D. Angelis, A. Moschitta, and P. Carbone, "Positioning techniques in indoor environments based on stochastic modeling of uwb round-trip-time measurements," IEEE Transactions on Intelligent Transportation Systems, vol. 17, no. 8, pp. 2272-2281, 2016.

[7] L. Zhao, A. M. Haimovich, "Performance of ultrawideband communications in the presence of interference," IEEE J. Select. Areas Commun., vol. 20, December 2002.

[8] K. Hamaguchi, H. Ogawa, T. Kobaysashi, R. Kohno, "Development of Ultra-Wideband short-range impulse radar system for vehicular applications", IEICE Trans, Electron., vol. E88-c, no. 10, pp. 1922-1931, 2005.

[9] A. Sorrentino, F. Nunziata, G. Ferrara, M. Migliaccio, "Reverberating Chamber Profile Identification", IET Microwaves, Antennas \& Propagation, vol.6, no.13, 1468-1472, 2012.

[10] G. Ferrara, M. Migliaccio, F. Nunziata, A. Sorrentino, "GK-based observation of metallic objects at sea in fullresolution SAR data: a multi-polarization study", IEEE Journal of Oceanic Engineering, vol 36, no2. 2011.

[11] A.Sorrentino, G.Ferrara, M.Migliaccio, "Kurtosis index to characterize near LOS conditions in reverberating chambers", IET Microwaves, Antennas \& Propagation, vol.7, no. 3, pp.175-179, 2013.

[12]B. Hardling, C. Tremblay, D. Cousineau, "Standard errors: a review and evaluation of standard error estimators using Monte Carlo simulations", Tutorials in Quantitative Methods for Psychology, vol. 10, no.2, pp. 107-123, 2014.

[13]B. Efron, R. J. Tibshirani, An Intorduction to the Bootstrap, Chpaman \& Hall/CRC, New York, NY.

[14] S. H. Han, J. H. Lee, “An Overview of Peak-to-Average Power Ratio Reduction Techniques for Multicarrier Transmission”, IEEE Wireless Communications, vol.12, no.2, pp.56-65, 2005.

[15] W. H. Press, S. A. Eukolsky, W. T. Vetterling, B. P. Flannery, Numerical Recies in C: The Art of Scientific Computating, Second Edition, Cambridge University Press, New York, NY, 2002.

[16] J. Kenney and E. S. Keeping, Mathematics of Statistics, van Nostrand, 1963. 